Proceedings of the Edinburgh Mathematical Society (2006) 49, 201-214 (C)

DOI:10.1017/S0013091504001427 Printed in the United Kingdom

\title{
ON THE BEHAVIOUR OF EIGENVALUES ASSOCIATED WITH STURM-LIOUVILLE PROBLEMS ON SHRINKING DOMAINS
}

\author{
D. J. NEEDHAM AND A. C. KING \\ Department of Mathematics, University of Reading, \\ PO Box 220, Reading RG6 6AX, UK (d.j.needham@reading.ac.uk)
}

(Received 27 November 2004)

\begin{abstract}
We establish results concerning the detailed asymptotic structure of the eigenvalues of a class of regular and singular Sturm-Liouville problems as the domain size shrinks to zero. This is motivated by the recent paper of Bailey et al. and provides an extensive generalization of the results therein.
\end{abstract}

Keywords: Sturm-Liouville eigenvalue problem; shrinking domains; asymptotic behaviour

2000 Mathematics subject classification: Primary 34B24

\section{Introduction}

In this paper we consider aspects of the classical Sturm-Liouville problem

$$
\left[p(X) Y_{X}\right]_{X}+[q(X)+r(X) \lambda] Y=0, \quad X \in(0, a),
$$

with conditions

$$
Y^{\prime}(0)=0 \quad(\text { when } p(0)>0)
$$

or

$$
Y(X), Y^{\prime}(X) \text { bounded as } X \rightarrow 0^{+} \quad(\text { when } p(0)=0),
$$

together with

$$
Y^{\prime}(a)=0,
$$

in the framework of the classical setting for Sturm-Liouville problems (as expounded in, for example, [2, Chapters 7,8$])$; we restrict our attention to $a \in(0,1]$. We will refer to this problem throughout as SL. In SL we assume (as in $[\mathbf{2}]$ ) that $p, q, r:[0,1] \rightarrow \mathbb{R}$ are independent of $a$ and $\lambda$, with $q, r \in C[0,1]$ and $p \in C^{1}[0,1]$. Moreover, we assume that

$$
r(X), p(X)>0 \quad \forall X \in(0,1],
$$


and that $p, q$ and $r$ are analytic at $X=0$ (that is, $\exists \delta>0$ such that $p, q$ and $r$ have convergent power-series representations on $[0, \delta)$ ). Thus we have

$$
\begin{aligned}
& r(X)=r_{0}+r_{S} X^{S}+o\left(X^{S}\right), \\
& q(X)=q_{0}+q_{N} X^{N}+o\left(X^{N}\right), \\
& p(X)=p_{0}+p_{1} X+o(X),
\end{aligned}
$$

as $X \rightarrow 0^{+}$, with $r_{0}, p_{0} \geqslant 0$ and $r_{S}, q_{N}, p_{1} \neq 0$, while $r_{S}>0$ when $r_{0}=0$ and $p_{1}>0$ when $p_{0}=0$. Here $S, N \in \mathbb{N}=\{1,2, \ldots\}$. With the conditions above, an application of the Frobenius method readily reveals that, when $p_{0}>0$, SL is regular, while, when $p_{0}=0$, SL is singular, with the endpoint $X=0$ being in the limit-circle non-oscillatory class. In particular, for any $\lambda \in \mathbb{C}$, let $Y_{+}$and $Y_{-}$be two linearly independent solutions to equation $(1.1)$ on $[0, a]$. In the case $p_{0}>0$, since $p, q, r$ are analytic at $X=0, Y_{+}, Y_{-}$ are analytic at $X=0$, and we may take

$$
\begin{array}{ll}
Y_{+}(0)=1, & Y_{+}^{\prime}(0)=0 \\
Y_{-}(0)=0, & Y_{-}^{\prime}(0)=1
\end{array}
$$

so boundary condition (1.2) is associated with the solution $Y_{+}$. In the case $p_{0}=0$ $\left(p_{1} \neq 0\right)$, the two linearly independent solutions $Y_{+}$and $Y_{-}$may be chosen so that $Y_{+}$is analytic at $X=0$ while $Y_{-}$is singular at $X=0$. Specifically, $Y_{+}$may be chosen so that

$$
Y_{+}(0)=1, \quad Y_{+}^{\prime}(0)=\frac{-\left(q_{0}+r_{0} \lambda\right)}{p_{1}},
$$

while $Y_{-}$has

$$
Y_{-}(X)=\log X+O(X \log X)
$$

as $X \rightarrow 0^{+}$. Thus, $Y_{+}$is the principal solution of equation (1.1) at $X=0$, and it is $Y_{+}$that is associated with boundary condition (1.3). Standard Sturm-Liouville theory (see below) determines that SL has a purely discrete spectrum, consisting of eigenvalues $\lambda=\lambda_{r}(a) \in \mathbb{R}$, for $r=0,1,2, \ldots$, with

$$
\lambda_{0}(a)<\lambda_{1}(a)<\lambda_{2}(a)<\cdots,
$$

and $\lambda_{r}(a) \rightarrow \infty$ as $r \rightarrow \infty$, for each $a>0$. The purpose of this paper is to obtain precise information about the asymptotic behaviour of the eigenvalues (1.7) as $a \rightarrow 0^{+}$, and this has been achieved in all cases for SL. We should point out at this stage that although we know of no previous results concerning the asymptotic behaviour of the eigenvalues of SL with shrinking domain size $a$, many papers have been published concerning the asymptotic structure of the eigenvalues of SL for fixed domain size $a$, as $r \rightarrow \infty$, in (1.7) (see, for example, $[\mathbf{3}]$ and $[\mathbf{4}]$ ). The problem of a two-dimensional domain shrinking to a one-dimensional domain has also been studied [5]. Here we are studying a one-dimensional domain shrinking to a point. Our results may be summarized in the following theorems.

Theorem 1.1 (regular case). Consider $S L$ with $p_{0}>0$. We then have the following. 
(a) $r_{0}>\mathbf{0}$. We have

$$
\lambda_{0}(a) \rightarrow-\frac{q_{0}}{r_{0}} \quad \text { as } a \rightarrow 0^{+},
$$

while

$$
\lambda_{r}(a) \sim \frac{p_{0} r^{2} \pi^{2}}{r_{0}} a^{-2} \quad \text { as } a \rightarrow 0^{+}
$$

for $r=1,2,3, \ldots$.

(b) $r_{0}=0$.

(i) For $q_{0} \neq 0$ we have

$$
\lambda_{0}(a) \rightarrow\left\{\begin{array}{ll}
-\infty, & q_{0}>0, \\
+\infty, & q_{0}<0,
\end{array} \quad \text { as } a \rightarrow 0^{+}\right.
$$

with $\lambda_{0}(a)=O\left(a^{-S}\right)$ as $a \rightarrow 0^{+}$.

(ii) For $q_{0}=0$ we have the following.

$$
\begin{aligned}
& N=S: \quad \lambda_{0}(a) \rightarrow-\frac{q_{N}}{r_{S}} \quad \text { as } a \rightarrow 0^{+} . \\
& N>S: \quad \lambda_{0}(a) \rightarrow 0 \quad \text { as } a \rightarrow 0^{+}, \\
& \text {with } \lambda_{0}(a)=O\left(a^{N-S}\right) \quad \text { as } a \rightarrow 0^{+} \text {. } \\
& N<S: \quad \lambda_{0}(a) \rightarrow\left\{\begin{array}{ll}
-\infty, & q_{N}>0, \\
+\infty, & q_{N}<0,
\end{array} \quad \text { as } a \rightarrow 0^{+},\right. \\
& \text {with } \lambda_{0}(a)=O\left(a^{-(S-N)}\right) \quad \text { as } a \rightarrow 0^{+} \text {. }
\end{aligned}
$$

Correspondingly, we have, in either of the cases (i) and (ii) above, that

$$
\lambda_{r}(a) \sim \frac{p_{0} \mu_{r}^{0}}{r_{S}} a^{-(2+S)} \quad \text { as } a \rightarrow 0^{+}
$$

for $r=1,2,3, \ldots$, where

$$
0=\mu_{0}^{0}<\mu_{1}^{0}<\mu_{2}^{0}<\cdots
$$

are the eigenvalues of the regular Sturm-Liouville problem

$$
\begin{gathered}
Y_{x x}+x^{S} \mu Y=0, \quad x \in(0,1), \\
Y_{x}(0)=Y_{x}(1)=0 .
\end{gathered}
$$

Theorem 1.2 (singular case). Consider $S L$ with $p_{0}=0$. Then, for $\lambda_{0}(a)$, we have the following.

(a) $\boldsymbol{q}_{\mathbf{0}} \neq \mathbf{0}, \boldsymbol{r}_{\mathbf{0}}>\mathbf{0} . \lambda_{0}(a) \rightarrow-\left(q_{0} / r_{0}\right)$ as $a \rightarrow 0^{+}$. 
(b) $\boldsymbol{q}_{\mathbf{0}}=\mathbf{0}, \boldsymbol{r}_{\mathbf{0}}>\mathbf{0}$. $\lambda_{0}(a) \rightarrow 0$ as $a \rightarrow 0^{+}$, with $\lambda_{0}(a)=O\left(a^{N}\right)$ as $a \rightarrow 0^{+}$.

(c) $q_{0} \neq 0, r_{0}=0$.

$$
\lambda_{0}(a) \rightarrow\left\{\begin{array}{ll}
+\infty, & q_{0}<0, \\
-\infty, & q_{0}>0,
\end{array} \quad \text { as } a \rightarrow 0^{+}, \quad \text { with } \lambda_{0}(a)=O\left(a^{-S}\right) \text { as } a \rightarrow 0^{+}\right.
$$

(d) $\boldsymbol{q}_{\mathbf{0}}=\mathbf{0}, \boldsymbol{r}_{\mathbf{0}}=\mathbf{0}$. There are three cases as follows.

$$
\begin{aligned}
& N=S: \quad \lambda_{0}(a) \rightarrow-\left(q_{N} / r_{S}\right) \quad \text { as } a \rightarrow 0^{+} . \\
& N>S: \quad \lambda_{0}(a) \rightarrow 0 \quad \text { as } a \rightarrow 0^{+}, \\
& \text {with } \lambda_{0}(a)=O\left(a^{N-S}\right) \quad \text { as } a \rightarrow 0^{+} \text {. } \\
& N<S: \quad \lambda_{0}(a) \rightarrow\left\{\begin{array}{ll}
+\infty, & q_{N}<0, \\
-\infty, & q_{N}>0,
\end{array} \quad \text { as } a \rightarrow 0^{+},\right. \\
& \text {with } \lambda_{0}(a)=O\left(a^{-(S-N)}\right) \quad \text { as } a \rightarrow 0^{+} .
\end{aligned}
$$

Theorem 1.3 (singular case). Consider SL with $p_{0}=0$. Then, for $\lambda_{r}(a), r=$ $1,2,3, \ldots$, we have the following.

(a) $\boldsymbol{r}_{\mathbf{0}}>\mathbf{0}$. $\lambda_{r}(a) \sim\left(\mu_{r}^{0} / r_{0}\right) a^{-1}$ as $a \rightarrow 0^{+}$, where $0=\mu_{0}^{0}<\mu_{1}^{0}<\mu_{2}^{0}<\cdots$ are the eigenvalues of the singular Sturm-Liouville problem

$$
\begin{gathered}
\left(x Y_{x}\right)_{x}+\mu Y=0, \quad x \in(0,1), \\
Y(x), Y^{\prime}(x) \text { bounded as } x \rightarrow 0^{+}, \\
Y^{\prime}(1)=0 .
\end{gathered}
$$

(b) $\boldsymbol{r}_{\mathbf{0}}=\mathbf{0} . \lambda_{r}(a) \sim\left(\mu_{r}^{0} / r_{S}\right) a^{-(1+S)}$ as a $\rightarrow 0^{+}$, where now $0=\mu_{0}^{0}<\mu_{1}^{0}<\mu_{2}^{0}<\cdots$ are the eigenvalues of the singular Sturm-Liouville problem

$$
\begin{gathered}
\left(x Y_{x}\right)_{x}+x^{S} \mu Y=0, \quad x \in(0,1), \\
Y(x), Y^{\prime}(x) \text { bounded as } x \rightarrow 0^{+}, \\
Y^{\prime}(1)=0 .
\end{gathered}
$$

The work in this paper was motivated by the recent paper [1] , in which the following singular Sturm-Liouville problem appeared from a problem arising in fuel cell dynamics, namely

$$
\left.\begin{array}{c}
{\left[X Y_{X}\right]_{X}+\left[X^{3}+X \lambda\right] Y=0, \quad X \in(0, a)} \\
Y(X), Y^{\prime}(X) \text { bounded as } X \rightarrow 0^{+} \\
Y^{\prime}(a)=0 .
\end{array}\right\}
$$

Of particular interest in that paper is the behaviour of the eigenvalues of $(\mathrm{P})$ as the domain size $a \rightarrow 0^{+}$. This problem is now a special case of Theorems 1.2 and 1.3 , with

$$
p_{0}=0, \quad p_{1}=1, \quad q_{0}=0, \quad q_{3}=1, \quad r_{0}=0, \quad r_{1}=1,
$$


and $S=1, N=3$. Application of Theorems 1.2 and 1.3 then immediately gives us that $\lambda_{0}(a) \rightarrow 0$ as $a \rightarrow 0^{+}$, with $\lambda_{0}(a)=O\left(a^{2}\right)$ as $a \rightarrow 0^{+}$and $\lambda_{r}(a) \sim \mu_{r}^{0} a^{-2}$ as $a \rightarrow 0^{+}$, where $r=1,2, \ldots$, and $0=\mu_{0}^{0}<\mu_{1}^{0}<\mu_{2}^{0}<\cdots$ are the eigenvalues of the Sturm-Liouville problem

$$
\begin{gathered}
{\left[x Y_{x}\right]_{x}+x \mu Y=0, \quad x \in(0,1),} \\
Y(x), Y^{\prime}(x) \text { bounded as } x \rightarrow 0^{+}, \\
Y^{\prime}(1)=0 .
\end{gathered}
$$

This reproduces and extends the results given in [1, Theorem 6.1]. The results in [1] established in the particular case of $(\mathrm{P})$ that $\lambda_{0}(a) \rightarrow 0$ as $a \rightarrow 0^{+}$, while $\lambda_{r}(a) \rightarrow+\infty$ as $a \rightarrow 0^{+}$, without the above order estimates.

Theorem 1.1 is established in $\S 2$, while Theorem 1.2 is established in $\S 3$.

\section{Regular Sturm-Liouville problems}

In this section we consider the regular Sturm-Liouville problem, with eigenvalue parameter $\lambda$,

$$
\begin{gathered}
{\left[p(X) Y_{X}\right]_{X}+[q(X)+r(X) \lambda] Y=0, \quad X \in(0, a),} \\
Y^{\prime}(0)=Y^{\prime}(a)=0,
\end{gathered}
$$

which we will henceforth refer to as RSL. In the above, $a>0$ is the domain size, and the functions $p, q, r:[0,1] \rightarrow \mathbb{R}$ are all continuous on $[0,1]$, with $p$ being differentiable on $[0,1]$. Moreover, for a regular Sturm-Liouville problem, we require

$$
\begin{array}{ll}
p(X)>0 & \forall X \in[0,1], \\
r(X)>0 & \forall X \in[0,1],
\end{array}
$$

and we assume the additional conditions laid down in $\S 1$. As usual, an eigenvalue of RSL is a number $\lambda \in \mathbb{C}$ such that there exists a non-trivial function $Y:[0, a] \rightarrow \mathbb{C}$ that classically solves RSL. $Y(X)$ is the eigenfunction of RSL associated with the eigenvalue $\lambda$. We recall, from the classical Sturm-Liouville theory (see, for example, [2, Chapter 8]), the following results for RSL with conditions (2.3), (2.4).

(i) The eigenvalues of RSL are all real, and there are an infinite number of eigenvalues, $\lambda_{0}(a), \lambda_{1}(a), \lambda_{2}(a), \ldots$, forming a monotone increasing sequence with $\lambda_{n}(a) \rightarrow \infty$ as $n \rightarrow \infty$.

(ii) Each eigenvalue $\lambda_{r}(a)$ has a unique eigenfunction $Y_{r}(\cdot, a):[0, a] \rightarrow \mathbb{R}$ such that $Y_{r}(0, a)>0, Y_{r}(a, a) \neq 0$ and

$$
\int_{0}^{a} r(X) Y_{r}^{2}(X, a) \mathrm{d} X=1
$$

All other eigenfunctions corresponding to the eigenvalue $\lambda_{r}(a)$ are simply complex scalar multiples of $Y_{r}(X, a)$. 
(iii) The eigenfunction $Y=Y_{r}(X, a)$ corresponding to the eigenvalue $\lambda_{r}(a)$ has exactly $r$ zeros in $(0, a)$.

We now wish to examine the behaviour of the eigenvalues $\lambda_{r}(a)(r=0,1,2, \ldots)$ of RSL as $a \rightarrow 0^{+}$. It is convenient to consider the cases $r(0)=0$ and $r(0)>0$ separately.

\subsection{The case $r(0)>0$}

In this case $r:[0,1] \rightarrow \mathbb{R}$ is bounded above zero, and we begin by considering the behaviour of $\lambda_{0}(a)$ as $a \rightarrow 0^{+}$. From (ii) and (iii) it follows immediately that

$$
I_{0}(a)=\int_{0}^{a} r(X) Y_{0}(X, a) \mathrm{d} X>0 .
$$

Now, via equation (2.1) we have

$$
\left[p(X) Y_{0 X}(X, a)\right]_{X}+\left[q(X)-\frac{q_{0}}{r_{0}} r(X)+r(X)\left(\lambda_{0}(a)+\frac{q_{0}}{r_{0}}\right)\right] Y_{0}(X, a)=0, \quad X \in(0, a),
$$

where $r_{0}=r(0)>0$ and $q_{0}=q(0)$. We next apply the operation $\int_{+\varepsilon}^{a-\varepsilon} \cdots \mathrm{d} X$, for some $\varepsilon>0$, to (2.6), which gives

$$
\begin{aligned}
& p(a-\varepsilon) Y_{0 X}(a-\varepsilon, a)-p(\varepsilon) Y_{0 X}(\varepsilon, a) \\
& \quad+\int_{\varepsilon}^{a-\varepsilon} Q(X) Y_{0}(X, a) \mathrm{d} X+\left(\lambda_{0}(a)+\frac{q_{0}}{r_{0}}\right) \int_{\varepsilon}^{a-\varepsilon} r(X) Y_{0}(X, a) \mathrm{d} X=0,
\end{aligned}
$$

where $Q:[0,1] \rightarrow \mathbb{R}$ is given by $Q(X)=q(X)-\left(q_{0} / r_{0}\right) r(X)$, which is continuous, and has

$$
Q(0)=0 .
$$

On letting $\varepsilon \rightarrow 0$, and using continuity, together with conditions (2.2), we obtain

$$
\left[\lambda_{0}(a)+\frac{q_{0}}{r_{0}}\right] \int_{0}^{a} r(X) Y_{0}(X, a) \mathrm{d} X+\int_{0}^{a} Q(X) Y_{0}(X, a) \mathrm{d} X=0 .
$$

A simple rearrangement of (2.8), using (2.5), leads us to

$$
\lambda_{0}(a)+\frac{q_{0}}{r_{0}}=-\frac{J_{0}(a)}{I_{0}(a)},
$$

where

$$
J_{0}(a)=\int_{0}^{a} Q(X) Y_{0}(X, a) \mathrm{d} X .
$$

Now, $Q:[0,1] \rightarrow \mathbb{R}$ is continuous, and we set

$$
\left.\begin{array}{l}
Q_{M}(a)=\max \{Q(X): X \in[0, a]\}, \\
Q_{m}(a)=\min \{Q(X): X \in[0, a]\},
\end{array}\right\}
$$


so that, since $Y_{0}(X, a)>0$ for all $X \in[0, a]$, we have

$$
-Q_{M}(a) K_{0}(a) \leqslant-J_{0}(a) \leqslant-Q_{m}(a) K_{0}(a),
$$

where

$$
K_{0}(a)=\int_{0}^{a} Y_{0}(X, a) \mathrm{d} X>0
$$

Therefore,

$$
-\left|Q_{M}(a)\right| K_{0}(a) \leqslant-J_{0}(a) \leqslant\left|Q_{m}(a)\right| K_{0}(a)
$$

which leads to

$$
-\frac{\left|Q_{M}(a)\right| K_{0}(a)}{I_{0}(a)} \leqslant-\frac{J_{0}(a)}{I_{0}(a)} \leqslant \frac{\left|Q_{m}(a)\right| K_{0}(a)}{I_{0}(a)} .
$$

Now, $r:[0,1] \rightarrow \mathbb{R}$ is continuous, and we set

$$
\left.\begin{array}{rl}
r_{M}(a) & =\max \{r(X): X \in[0, a]\}>0, \\
r_{m}(a) & =\min \{r(X): X \in[0, a]\}>0,
\end{array}\right\}
$$

as $r(X)>0$ for $X \in[0,1]$. Thus

$$
r_{m}(a) K_{0}(a) \leqslant I_{0}(a) \leqslant r_{M}(a) K_{0}(a),
$$

and so (2.15) leads to

$$
-\frac{\left|Q_{M}(a)\right|}{r_{m}(a)} \leqslant-\frac{J_{0}(a)}{I_{0}(a)} \leqslant \frac{\left|Q_{m}(a)\right|}{r_{m}(a)}
$$

which gives, via (2.9),

$$
-\frac{\left|Q_{M}(a)\right|}{r_{m}(a)}-\frac{q_{0}}{r_{0}} \leqslant \lambda_{0}(a) \leqslant \frac{\left|Q_{m}(a)\right|}{r_{m}(a)}-\frac{q_{0}}{r_{0}} .
$$

Now, as $a \rightarrow 0^{+}$, we observe that

$$
r_{m}(a) \rightarrow r_{0}>0,
$$

while

$$
\left.\begin{array}{rl}
Q_{M}(a) \rightarrow Q(0) & =0, \\
Q_{m}(a) \rightarrow Q(0) & =0
\end{array}\right\}
$$

on using the continuity of $r$ and $Q$. Therefore, using (2.19) and (2.20), and allowing $a \rightarrow 0^{+}$in (2.18), we finally have

$$
\lambda_{0}(a) \rightarrow-\frac{q_{0}}{r_{0}} \quad \text { as } a \rightarrow 0^{+} .
$$

Next we consider the behaviour of $\lambda_{r}(a)(r=1,2, \ldots)$ as $a \rightarrow 0^{+}$. We cannot follow the previous approach for $\lambda_{0}(a)$, since in this case we cannot guarantee that

$$
\int_{0}^{a} r(X) Y_{r}(X, a) \mathrm{d} X \neq 0
$$


as was the case before. To proceed, we consider the modified RSL

$$
\begin{gathered}
{\left[p(a x) Y_{x}\right]_{x}+\left[a^{2} q(a x)+r(a x) \mu\right] Y=0, \quad x \in(0,1),} \\
Y_{x}(0)=Y_{x}(1)=0,
\end{gathered}
$$

where $a \in[0,1]$ and $\mu$ is now the eigenvalue parameter. We refer to this regular SturmLiouville problem as RSL'. In view of the properties on $p(X), q(X)$ and $r(X)$ for $X \in$ $[0,1]$, as laid down in $\S 1$, it follows that statements (i)-(iii) apply to RSL' for each $a \in$ $[0,1]$. Let $a \in[0,1]$, say, and denote the eigenvalues of $\mathrm{RSL}^{\prime}$ as $\mu=\mu_{r}(a)(r=0,1,2, \ldots)$, where

$$
\mu_{0}(a)<\mu_{1}(a)<\mu_{2}(a)<\cdots .
$$

Now let $Y^{+}(x, a, \mu), x \in[0,1]$, be that solution to equation (2.22) which has

$$
Y^{+}(0, a, \mu)=1, \quad Y_{x}^{+}(0, a, \mu)=0 .
$$

The eigenvalues of $\mathrm{RSL}^{\prime}$ at fixed $a \in[0,1]$ are then those values of $\mu \in \mathbb{R}$ such that

$$
\phi(a, \mu) \equiv Y_{x}^{+}(1, a, \mu)=0 .
$$

Now, for $a$ sufficiently small - in fact, $a \in\left[0, \frac{1}{2} \delta\right]$, with $\delta>0$ as defined in $\S 1$ - the coefficients of equation (2.22) are analytic functions of $(x, a, \mu) \in[0,1] \times\left[0, \frac{1}{2} \delta\right] \times \mathbb{R}$, following the conditions on $p, q$ and $r$ as laid down in $\S 1$. It follows immediately from [2, Chapter 1, p. 36, Theorem 8.3] that $Y^{+}(x, a, \mu)$, and hence $Y_{x}^{+}(x, a, \mu)$, are analytic functions of $(x, a, \mu) \in[0,1] \times\left[0, \frac{1}{2} \delta\right] \times \mathbb{R}$. Hence $\phi(a, \mu)$ is an analytic function of $(a, \mu) \in\left[0, \frac{1}{2} \delta\right] \times \mathbb{R}$. Now suppose that $\left(a^{*}, \mu^{*}\right) \in\left[0, \frac{1}{2} \delta\right] \times \mathbb{R}$ is such that $\phi\left(a^{*}, \mu^{*}\right)=0$, then it follows from (iii) in $\S 2$ that $\phi_{\mu}\left(a^{*}, \mu^{*}\right) \neq 0$. It is now a consequence of the implicit function theorem that $\phi(a, \mu)=0$ defines a unique differentiable function $\mu=f(a)$ in a neighbourhood of $a=a^{*}$, and such that $f\left(a^{*}\right)=\mu^{*}$. We conclude that the eigenvalues of RSL' depend continuously (in fact, differentiably) on $a$ at each $a \in\left[0, \frac{1}{2} \delta\right]$. Thus, for each $r=0,1,2, \ldots$,

$$
\mu_{r}:\left[0, \frac{1}{2} \delta\right] \rightarrow \mathbb{R}
$$

is continuous. Now, if we set $a=0$ in $\mathrm{RSL}^{\prime}$, it is trivial to establish that

$$
\mu_{r}(0)=\frac{p_{0}}{r_{0}} r^{2} \pi^{2}, \quad r=0,1,2, \ldots
$$

However, $\mu_{r}(a)$ is continuous for $a \in\left[0, \frac{1}{2} \delta\right]$, hence

$$
\mu_{r}(a) \rightarrow \frac{p_{0}}{r_{0}} r^{2} \pi^{2} \quad \text { as } a \rightarrow 0^{+},
$$

for each $r=0,1,2, \ldots$. We now establish the relationship between the eigenvalues of RSL and the eigenvalues of RSL' for each $a \in(0,1]$, via the following lemma.

Lemma 2.1. For each $a \in(0,1]$ the eigenvalues of $R S L$ and $R S L^{\prime}$ are related by $\mu_{r}(a)=a^{2} \lambda_{r}(a)$ for each $r=0,1,2, \ldots$. 
Proof. On making the transformation $x=a^{-1} X, \mu=a^{2} \lambda$ (which is invertible for $a \in(0,1])$, we observe that $\mathrm{RSL}^{\prime}$ is transformed into RSL.

Thus, using (2.27) together with the above Lemma 2.1 we have

$$
a^{2} \lambda_{r}(a) \rightarrow \frac{p_{0}}{r_{0}} r^{2} \pi^{2} \quad \text { as } a \rightarrow 0^{+}
$$

for each $r=0,1,2, \ldots$ For $r=0,(2.28)$ tells us $\lambda_{0}(a)=o\left(a^{-2}\right)$ as $a \rightarrow 0^{+}$.

However, we have already obtained a better estimate for $\lambda_{0}(a)$ as $a \rightarrow 0^{+}$in (2.21). For $r=1,2,3, \ldots,(2.28)$ gives us the precise estimate that

$$
\lambda_{r}(a) \sim \frac{p_{0} r^{2} \pi^{2}}{r_{0}} a^{-2} \text { as } a \rightarrow 0^{+},
$$

and so, in particular,

$$
\lambda_{r}(a) \rightarrow+\infty \quad \text { as } a \rightarrow 0^{+},
$$

for each $r=1,2,3, \ldots$. This completes the analysis for this case.

\subsection{The case $r(0)=0$}

We consider RSL with $r(0)=0$. We first observe that statements (i)-(iii) still hold for RSL when $r(0)=0$ (see, for example, $[\mathbf{2}$, Chapter $8, \S 2]$ ). In addition, we will assume, in this case, that, as laid out in $\S 1$,

(C1) $p(X)=p_{0}+p_{1} X+o(X)$,

(C2) $q(X)=q_{0}+q_{N} X^{N}+o\left(X^{N}\right)$,

(C3) $r(X)=r_{S} X^{S}+o\left(X^{S}\right)$

as $X \rightarrow 0$, with $p_{0}>0, r_{S}>0$ and $q_{N} \neq 0$, for some $S, N \in \mathbb{N}$.

Following precisely the same arguments as in the previous case, we arrive at

$$
\lambda_{0}(a)=-\frac{\int_{0}^{a} q(X) Y_{0}(X, a) \mathrm{d} X}{\int_{0}^{a} r(X) Y_{0}(X, a) \mathrm{d} X} .
$$

Hence, in terms of our previous notation,

$$
-\frac{q_{M}(a) K_{0}(a)}{I_{0}(a)} \leqslant \lambda_{0}(a) \leqslant-\frac{q_{m}(a) K_{0}(a)}{I_{0}(a)},
$$

where

$$
\left.\begin{array}{l}
q_{M}(a)=\max \{q(X): X \in[0, a]\}, \\
q_{m}(a)=\min \{q(X): X \in[0, a]\},
\end{array}\right\}
$$

and we recall that

$$
q_{M}(a), q_{m}(a) \rightarrow q_{0}
$$


as $a \rightarrow 0^{+}$. Also, we have

$$
\begin{aligned}
I_{0}(a) & =\int_{0}^{a} r(X) Y_{0}(X, a) \mathrm{d} X \\
& =a \int_{0}^{1} r(a w) Y_{0}(a w, a) \mathrm{d} w \\
& \sim r_{S} a^{1+S} L_{0}^{S}(a) \quad \text { as } a \rightarrow 0^{+},
\end{aligned}
$$

where, via (C3),

$$
L_{0}^{S}(a)=\int_{0}^{1} w^{S} Y_{0}(a w, a) \mathrm{d} w
$$

In addition, we note that

$$
K_{0}(a)=a \int_{0}^{1} Y_{0}(a w, a) \mathrm{d} w
$$

so that

$$
\frac{K_{0}(a)}{I_{0}(a)} \sim \frac{a L_{0}^{0}(a)}{r_{S} a^{1+S} L_{0}^{S}(a)} \quad \text { as } a \rightarrow 0^{+} .
$$

Now,

$$
\frac{L_{0}^{0}(a)}{L_{0}^{S}(a)}=\frac{\int_{0}^{1} Y_{0}(a w, a) \mathrm{d} w}{\int_{0}^{1} w^{S} Y_{0}(a w, a) \mathrm{d} w}=\phi_{S}(a),
$$

and it follows that, for any $S=1,2, \ldots$,

$$
\phi_{S}(a)>1 \text { for each } a>0 .
$$

Therefore, we have

$$
\frac{K_{0}(a)}{I_{0}(a)} \sim \frac{1}{r_{S}} a^{-S} \phi_{S}(a) \rightarrow+\infty
$$

as $a \rightarrow 0^{+}$, via (2.37) and (2.38). There are now two cases to consider.

(1) $\boldsymbol{q}_{\mathbf{0}} \neq \mathbf{0}$. With $q_{0} \neq 0$ we have, via (2.32), (2.34) and (2.39), that

$$
\lambda_{0}(a) \sim-\frac{q_{0}}{r_{S}} a^{-S} \phi_{S}(a) \rightarrow \begin{cases}-\infty, & q_{0}>0 \\ +\infty, & q_{0}<0\end{cases}
$$

as $a \rightarrow 0^{+}$.

(2) $\boldsymbol{q}_{\mathbf{0}}=\mathbf{0}$. With $q_{0}=0$ we have

$$
\int_{0}^{a} q(X) Y_{0}(X, a) \mathrm{d} X=a \int_{0}^{1} q(a w) Y_{0}(a w, a) \mathrm{d} w \sim q_{N} a^{1+N} L_{0}^{N}(a),
$$

as $a \rightarrow 0^{+}$, via (C2). Hence, using (2.31) with (2.35), (2.41) and (2.38) we obtain

$$
\lambda_{0}(a) \sim-\frac{q_{N}}{r_{S}} a^{N-S} \frac{L_{0}^{N}(a)}{L_{0}^{S}(a)},
$$

as $a \rightarrow 0^{+}$. There are three subcases to consider. 
(a) $N=S$. In this case we have, directly from (2.42),

$$
\lambda_{0}(a) \rightarrow-\frac{q_{S}}{r_{S}} \quad \text { as } a \rightarrow 0^{+} .
$$

(b) $N>S$. In this case

$$
0<\frac{L_{0}^{N}(a)}{L_{0}^{S}(a)}<1
$$

for all $a>0$. Thus, via (2.42),

$$
\lambda_{0}(a)=O\left(a^{N-S}\right) \rightarrow 0 \quad \text { as } a \rightarrow 0^{+} .
$$

(c) $N<S$. In this case

$$
\frac{L_{0}^{N}(a)}{L_{0}^{S}(a)}>1
$$

for all $a>0$. Hence, via (2.42),

$$
\lambda_{0}(a) \rightarrow \begin{cases}-\infty, & q_{N}>0, \\ +\infty, & q_{N}<0\end{cases}
$$

as $a \rightarrow 0^{+}$.

The analysis is now complete for $\lambda_{0}(a)$. We next consider the behaviour of $\lambda_{r}(a)$ $(r=1,2, \ldots)$ as $a \rightarrow 0^{+}$. We proceed as in the previous section. We consider the modified RSL

$$
\begin{gathered}
{\left[\frac{p(a x)}{p_{0}} Y_{x}\right]_{x}+\left[\frac{a^{2} q(a x)}{p_{0}}+\frac{r(a x)}{r_{S} a^{S}} \mu\right] Y=0, \quad x \in(0,1),} \\
Y_{x}(0)=Y_{x}(1)=0,
\end{gathered}
$$

where $a \in[0,1]$ and $\mu$ is now the eigenvalue parameter. We refer to this regular SturmLiouville problem as RSL". Since $r(a x) a^{-S}$ is continuous on $(a, x) \in[0,1] \times[0,1]$, via (C3), statements (i)-(iii) apply to RSL" for each $a \in[0,1]$, and we denote the eigenvalues of RSL" by $\mu=\mu_{r}(a)(r=0,1,2, \ldots)$, where $\mu_{0}(a)<\mu_{1}(a)<\mu_{2}(a)<\cdots$.

Also, since $p(a x), a^{2} q(a x)$ and $r(a x) a^{-S}$ (removable singularity at $a=0$ ) are analytic for $(x, a) \in[0,1] \times\left[0, \frac{1}{2} \delta\right]$, we can conclude as before that, for each $r=0,1,2, \ldots$, $\mu_{r}:\left[0, \frac{1}{2} \delta\right] \rightarrow \mathbb{R}$ is continuous. Now let $a \rightarrow 0^{+}$in $\mathrm{RSL}^{\prime \prime}$, and we obtain

$$
\left.\begin{array}{c}
Y_{x x}+x^{S} \mu Y=0, \quad x \in(0,1), \\
Y_{x}(0)=Y_{x}(1)=0 .
\end{array}\right\}
$$

This is a regular Sturm-Liouville problem, now independent of $a$. Observe that (RSL0) has eigenvalue $\mu=0$ with associated eigenfunction $Y(x) \equiv 1$ for $x \in[0,1]$. On using statement (iii) we can conclude that $\mu=0$ is the lowest eigenvalue of (RSL0). We denote the eigenvalues of (RSL0) by $\mu_{r}^{0}, r=0,1,2, \ldots$, with

$$
0=\mu_{0}^{0}<\mu_{1}^{0}<\mu_{2}^{0}<\cdots,
$$


and we conclude that

$$
\mu_{r}(0)=\mu_{r}^{0} \quad \text { for each } r=0,1,2, \ldots,
$$

and so

$$
\mu_{r}(a) \rightarrow \mu_{r}^{0} \quad \text { as } a \rightarrow 0^{+},
$$

for each $r=0,1,2, \ldots$ We now relate the eigenvalues of RSL to those of RSL" for each $a \in(0,1]$ via the following lemma.

Lemma 2.2. For each $a \in(0,1]$ the eigenvalues of $R S L$ and $R S L^{\prime \prime}$ are related by $\mu_{r}(a)=\left(r_{S} / p_{0}\right) a^{2+S} \lambda_{r}(a)$ for each $r=0,1,2, \ldots$

Proof. On making the transformation $x=a^{-1} X, \mu=\left(r_{S} / p_{0}\right) a^{2+S} \lambda$ (which is invertible for each $a \in(0,1])$ we observe that RSL" is transformed to RSL.

Hence, on using (2.50) together with Lemma 2.2 we have $\left(r_{S} / p_{0}\right) a^{2+S} \lambda_{r}(a) \rightarrow \mu_{r}^{0}$ as $a \rightarrow 0^{+}$, for each $r=0,1,2, \ldots$, and so

$$
\lambda_{r}(a) \sim \frac{p_{0} \mu_{r}^{0}}{r_{S}} a^{-(2+S)} \quad \text { as } a \rightarrow 0^{+},
$$

for each $r=1,2,3, \ldots$ In particular, then,

$$
\lambda_{r}(a) \rightarrow+\infty \quad \text { as } a \rightarrow 0^{+},
$$

for $r=1,2,3, \ldots$ This completes the analysis for this case.

\section{Remarks on the singular Sturm-Liouville problems}

In this section we consider the Sturm-Liouville problem (2.1), (2.2) with all of the additional conditions applying, except that we replace condition (2.3) by

$$
p(X)>0 \quad \forall X \in(0,1]
$$

with

$$
p(0)=0
$$

and

$$
p(X)=p_{1} X+o(X) \quad \text { as } X \rightarrow 0,
$$

where $p_{1}>0$ and the first of boundary conditions (2.2) is replaced by $Y(X), Y^{\prime}(X)$ bounded as $X \rightarrow 0^{+}$.

This is referred to as a singular Sturm-Liouville problem, and we will henceforth refer to it as SSL. We observe that statements (i)-(iii) still apply to SSL as the singularity at $X=0$ in equation (2.1) is in the limit-circle non-oscillatory class (see $\S 1$ ). We again wish to examine the behaviour of the eigenvalues $\lambda=\lambda_{r}(a)(r=0,1,2, \ldots)$ of SSL as $a \rightarrow 0^{+}$, and the theory can be developed immediately by following the approach of $\S 2.2$. Consequently, we do not reproduce details in this section but briefly quote 
the corresponding results. We note that the analyticity condition required to establish continuity of the eigenvalues of the corresponding scaled problem for $a \in\left[0, \frac{1}{2} \delta\right]$ follows in this case as we are considering the principal solution of the scaled equation on $[0,1]$, which is analytic for $(x, a, \mu) \in[0,1] \times\left[0, \frac{1}{2} \delta\right] \times \mathbb{R}$. For the lowest eigenvalue we have four cases as follows.

(a) $q_{0} \neq 0, r_{0}>0$. Here

$$
\lambda_{0}(a) \rightarrow-\frac{q_{0}}{r_{0}} \quad \text { as } a \rightarrow 0^{+} .
$$

(b) $q_{0}=0, r_{0}>0$. Here

$$
\lambda_{0}(a) \sim-\frac{q_{N}}{r_{0}} a^{N} \frac{L_{0}^{N}(a)}{L_{0}^{0}(a)}
$$

as $a \rightarrow 0^{+}$. Since

$$
0<\frac{L_{0}^{N}(a)}{L_{0}^{0}(a)}<1
$$

for all $a \in(0,1]$, we have

$$
\lambda_{0}(a) \rightarrow 0 \quad \text { as } a \rightarrow 0^{+} .
$$

(c) $q_{0} \neq 0, r_{0}=0$. In this case

$$
\lambda_{0}(a) \sim-\frac{q_{0}}{r_{S}} a^{-S} \frac{L_{0}^{0}(a)}{L_{0}^{S}(a)},
$$

as $a \rightarrow 0^{+}$, and

$$
\frac{L_{0}^{0}(a)}{L_{0}^{S}(a)}>1
$$

for all $a \in(0,1]$. Thus we have

$$
\lambda_{0}(a) \rightarrow \begin{cases}+\infty, & q_{0}<0, \\ -\infty, & q_{0}>0,\end{cases}
$$

as $a \rightarrow 0^{+}$.

(d) $q_{0}=r_{0}=0$. Here

$$
\lambda_{0}(a) \sim-\frac{q_{N}}{r_{S}} a^{N-S} \frac{L_{0}^{N}(a)}{L_{0}^{S}(a)},
$$

as $a \rightarrow 0^{+}$. Thus there are three subcases to consider as follows.

$$
\begin{array}{lll}
N=S: & \lambda_{0}(a) \rightarrow-\frac{q_{N}}{r_{S}} & \text { as } a \rightarrow 0^{+} . \\
N>S: & \lambda_{0}(a) \rightarrow 0 & \text { as } a \rightarrow 0^{+} . \\
N<S: & \lambda_{0}(a) \rightarrow\left\{\begin{array}{ll}
+\infty, & q_{N}<0, \\
-\infty, & q_{N}>0,
\end{array} \text { as } a \rightarrow 0^{+} .\right.
\end{array}
$$


For the higher eigenvalues, we obtain the following results.

(a) $r_{0}>\mathbf{0}$. In this case, we have

$$
\lambda_{r}(a) \sim \frac{\mu_{r}^{0}}{r_{0}} a^{-1} \quad \text { as } a \rightarrow 0^{+},
$$

for $r=1,2, \ldots$, where $0=\mu_{0}^{0}<\mu_{1}^{0}<\mu_{2}^{0}<\cdots$ are the eigenvalues of the singular Sturm-Liouville problem

$$
\begin{gathered}
\left(x Y_{x}\right)_{x}+\mu Y=0, \quad x \in(0,1), \\
Y(x), Y^{\prime}(x) \text { bounded as } x \rightarrow 0^{+}, \\
Y^{\prime}(1)=0 .
\end{gathered}
$$

(b) $r_{0}=\mathbf{0}$. Here we have

$$
\lambda_{r}(a) \sim \frac{\mu_{r}^{0}}{r_{S}} a^{-(1+S)} \quad \text { as } a \rightarrow 0^{+},
$$

for $r=1,2, \ldots$, where now $0=\mu_{0}^{0}<\mu_{1}^{0}<\mu_{2}^{0}<\cdots$ are the eigenvalues of the singular Sturm-Liouville problem

$$
\begin{gathered}
\left(x Y_{x}\right)_{x}+x^{S} \mu Y=0, \quad x \in(0,1), \\
Y(x), Y^{\prime}(x) \text { bounded as } x \rightarrow 0^{+}, \\
Y^{\prime}(1)=0 .
\end{gathered}
$$

All of our results have now been established, and the proofs of Theorems 1.1-1.3 are complete.

\section{References}

1. P. B. Bailey, J. Billingham, R. J. Cooper, W. N. Everitt, A. C. King, Q. Kong, H. Wu And A. Zettu, Eigenvalue problems in fuel cell dynamics, Proc. R. Soc. Lond. A 459 (2003), 241-261.

2. E. A. CodDington And N. Levinson, Theory of ordinary differential equations (McGraw-Hill, New York, 1955).

3. B. J. HARris, Asymptotics of eigenvalues for regular Sturm-Liouville problems, J. Math. Analysis Applic. 183 (1994), 25-36.

4. B. J. Harris and D. RACE, Asymptotics of eigenvalues for Sturm-Liouville problems with an interior singularity, J. Diff. Eqns 116(1) (1995), 88-118.

5. A. G. RAmm, Limit of spectra of the interior Neumann problems when a solid domain shrinks to a plane one, J. Math. Analysis Applic. 108 (1985), 107-112. 